\title{
L'intégration des dispositifs délibératifs dans les pratiques intercommunales
}

Hakim Akrab

\section{(2) OpenEdition}

1 Journals

Édition électronique

URL : http://journals.openedition.org/communicationorganisation/3725

DOI : 10.4000/communicationorganisation.3725

ISSN : $1775-3546$

Éditeur

Presses universitaires de Bordeaux

Édition imprimée

Date de publication : 1 juin 2012

Pagination : $51-61$

ISBN : 978-2-86781-821-9

ISSN : $1168-5549$

Référence électronique

Hakim Akrab, "L'intégration des dispositifs délibératifs dans les pratiques intercommunales », Communication et organisation [En ligne], 41 | 2012, mis en ligne le 01 juin 2015, consulté le 20 avril 2019. URL : http://journals.openedition.org/communicationorganisation/3725 ; DOI : 10.4000/ communicationorganisation.3725 


\title{
L'intégration des dispositifs délibératifs dans les pratiques intercommunales
}

\author{
Hakim Akrab'
}

Notre recherche doctorale porte sur l'inscription des Techniques d'Information et de Communication (Tic) dans la construction du territoire intercommunal. Nous analysons en particulier les pratiques des établissements intercommunaux dans la production et la diffusion de l'information. Notre méthode d'enquête repose sur l'analyse approfondie de cinq Établissements Publics de Coopération Intercommunale de la région Rhône-Alpes ${ }^{2}$ durant la période 2007-2011. Nous avons appliqué une grille d'analyse détaillée portant sur l'activité communicationnelle de chacun d'entre eux et conduit une trentaine d'entretiens en direction d'acteurs intercommunaux : professionnels de la communication intercommunale et responsables politiques. À travers cet article, notre objectif est de démontrer que l'intégration des dispositifs délibératifs au niveau des établissements intercommunaux est associée à des enjeux complexes qui articulent stratégies politiques, changements organisationnels et spécialisation des compétences. Dans un premier point, nous prendrons l'exemple des forums de discussions sur les sites web intercommunaux pour pointer l'intrusion des élus locaux dans la gestion de ces dispositifs techniques les intégrant à des stratégies politiques. Ensuite, nous évoquerons le conflit entre une association locale et la Communauté d'Agglomération Grenoble Alpes Métropole lors de la construction du stade d'agglomération afin de souligner les contournements de la législation dans l'application des dispositifs délibératifs. Enfin, il sera question d'étudier en détail l'appropriation de méthodes innovantes issues du milieu associatif dans l'organisation de la délibération à l'échelle intercommunale.

1 Hakim Akrab est docteur en sciences de l'information et de la communication au sein du laboratoire Groupe de Recherche sur les Enjeux de la Communication de l'Université Stendhal de Grenoble ; hakimakrab@voila.fr 2 La Communauté d'Agglomération Grenoble Alpes Métropole, la Communauté Urbaine du Grand Lyon, la Communauté de Communes du Pays Voironnais, la Communauté d'Agglomération Chambéry Métropole et la Communauté de Communes d'Oyonnax. 


\section{Les sites internet intercommunaux : l'échec des forums de discussions}

Les discours techno-déterministes relayés par l'État sur l'avènement d'une « cyberdémocratie » ont incité les pouvoirs locaux à développer des sites internet. Cette «injonction technologique ${ }^{3}$ » a souvent masquél'importance des moyens et compétences à mobiliser pour assurer leur pérennité. L'expérience des forums de discussions sur les sites intercommunaux et municipaux éclaire tout de même sur l'évolution organisationnelle des institutions territoriales. Les Tic ont accompagné une ouverture informationnelle en direction des habitants. Néanmoins, peu de collectivités territoriales ont réussi à pérenniser les forums à travers leurs sites internet. Lors d'un entretien avec un chargé de communication au sein de la Communauté de Communes du Pays Voironnais, nous avons constaté que ces outils donnent aux dirigeants politiques une meilleure appréciation des prises de positions de l'opinion locale : « Ce sont des supports efficaces dépendant des thématiques. Par exemple, pour le centre de stockage des déchets, la consultation des habitants sur internet a permis d'abandonner ce projet, les élus ont décidé de suivre l'opinion dominante chez les habitants pour s'orienter vers une autre solution qui est la destruction d'un maximum des déchets pour éviter la présence d'un centre de stockage. Plus de huit cents contributions sur le site. Le forum fonctionne bien mais il est plus un outil de la démocratie locale, parmi d'autres, utilisé de façon occasionnelle $e^{4}$ "

La rubrique «Démocratie locale » du site de la communauté du Pays Voironnais propose trois services : le forum avec différents sujets thématiques, la possibilité d'adresser un message aux responsables de services et enfin accéder aux comptes rendus de réunions. Concernant le forum, il s'agit plutôt de contributions où les habitants s'expriment sans que les échanges ne soient véritablement encadrés. Les élus intercommunaux ne prenant pas part aux débats, il semble difficile de les envisager comme le lieu de véritables discussions délibératives articulées à des prises de décisions. En effet, il convient de s'interroger sur les traces de ces échanges : sont-ils transmis aux dirigeants politiques? Sans l'élaboration d'une politique dans le domaine de la participation, à l'image des communautés ayant défini une charte de la participation, ces outils ne bénéficient pas d'une articulation officielle aux processus décisionnels. Les chartes de la participation ont procédé à un décloisonnement de l'information au niveau des services en imposant de nouvelles procédures comme la vulgarisation des documents techniques. Cependant, dans ses travaux sur l'usage des Tic dans les organisations, Alain Rallet souligne " que si des transformations organisationnelles sont directement associées aux nouvelles techniques, ce n'est pas que celles-ci induisent de nouveaux comportements mais elles concourent à rendre

3 MIÈGE B., La société conquise par la communication, III. Les Tic entre innovation technique et ancrage social, Grenoble, PUG, 2007, p. 22.

4 Entretien réalisé le 13 janvier 2010 avec le chargé de communication au sein de la Communauté d'Agglomération du Pays Voironnais. 
obsolète le mode ancien et obligent les entreprises à penser, à inventer les modes d'organisation qui rendent efficaces ces nouvelles techniques ${ }^{5}$ ». Ainsi, les Tic ont accompagné l'évolution organisationnelle des institutions territoriales dans la production et la diffusion de l'information. Elles ont favorisé l'émergence d'un modèle participatif qui, en apparence, intègre les agents et partenaires dans la définition d'un fonctionnement organisationnel comme le relève Christiane Demers ${ }^{6}$.

En outre, l'exemple des forums démontre que les citoyens, sans l'appui de structures organisées, constituent une diversité d'opinions qui ne peut pas représenter un pouvoir d'influence face à l'autorité politique des pouvoirs locaux. Ces outils ont également procédé à un changement dans l'exercice politique. Les dispositifs techniques permettent aux dirigeants locaux de prendre connaissance de l'opinion locale et d'adapter leur politique à ses positions. Il s'agit de nouvelles procédures qui sollicitent une compétence communicationnelle mais aussi une représentation différente de l'action politique. La recherche du consensus s'exerce selon un objectif que l'on peut définir comme une négociation des « intérêts ». Les individus expriment leurs positions sur le forum ou votent (sondages) de façon très diverse. Le suivi de ces échanges offre la possibilité d'avoir un aperçu des différentes tendances de l'opinion, sans qu'une réelle discussion ou que des informations sur les projets ne soient diffusées aux habitants. Dans ce cas de figure, il convient de remarquer que ces techniques ne concentrent pas une activité délibérative mais sont des moyens pour sonder les habitants. Le cas de l'intercommunalité dévoile donc une instrumentalisation de ces outils techniques. Ils furent souvent mis en ligne sans qu'en amont les dirigeants politiques n'amorcent les conditions nécessaires : diffusion de l'information et transparence des processus décisionnels. Cela nous oriente vers l'idée que les pratiques communicationnelles des structures intercommunales sont souvent détachées des logiques de fonctionnement de l'institution. La présence des dispositifs de délibération n'a pas induit une nouvelle gestion de l'action intercommunale ou l'intégration de la parole citoyenne dans son fonctionnement institutionnel. Comme le souligne Michèle Lacoste ${ }^{7}$ les pratiques que génèrent les Tic au sein des organisations ne peuvent pas être totalement prévisibles. Elles sont soumises à des glissements et détournements. Ces techniques ont eu une forte influence sur la gestion politique des projets. Elles ont permis un pilotage des actions à court terme, comme le souligne l'exemple de la communauté du Pays Voironnais. L'action locale s'est longtemps articulée à une temporalité politique reposant sur la durée des mandats de personnalités locales. Celles-ci

5 RALLET A., MUSSO P., Stratégies de communication et territoires, Paris, L’Harmattan, 1995, p. 123.

6 DEMERS Ch., «La diffusion d'un changement radical : un processus de redéfinition et de restructuration de l'organisation ", Communication E Organisation, 1993, n³, p. 44.

7 LACOSTE M., "Les objets et le travail en collectif ", dans Delcambre Pierre (dir.), Communications organisationnelles, objet, pratiques, dispositifs, Rennes, PUR, 2000, p. 73. 
étaient porteuses de l'identité du territoire politique qu'elles géraient. Depuis quelques années, comme le note Stéphane $\mathrm{Cadiou}^{8}$, la professionnalisation du métier d'élu local, notamment dans les villes moyennes et métropoles, a apporté des méthodes de gestion tournées vers la recherche d'un consensus avec les habitants. Les prises de décisions tentent, systématiquement, d'associer une diversité d'acteurs et l'opinion des citoyens. Dans cette configuration, la relation entre élus et citoyens est sans cesse redéfinie dans les différents projets incluant une confrontation. Au niveau organisationnel, les dirigeants politiques incitent les services à adopter des procédures nouvelles de diffusion de l'information liées à l'objectif de transparence des activités institutionnelles.

\section{La délibération au niveau intercommunal : l'exemple des enquêtes publiques}

La clarification des dispositions juridiques (loi Chevènement de 1999 et loi du 16 décembre 2010) concernant la constitution des communautés a accéléré le développement de l'intercommunalité au niveau national. Dès lors, nous observons que l'élargissement des compétences transférables a provoqué un déplacement des centres décisionnels au niveau local, passant de la municipalité à l'établissement intercommunal. Les procédures décisionnelles se sont aussi complexifiées. Loïc Blondiaux fait l'analyse de ce changement social : "Aujourd'bui les décisions les plus importantes se prennent à un niveau de l'échelon intercommunal où la concertation se pratique de manière pour le moins inégale selon les lieux. Les structures intercommunales ne sont pas, on le sait, élues au suffrage universel direct et les conseils de développement censés représenter la "société civile" auprès du pouvoir d'agglomération n'ont ni attributions ni moyens fixés par la loi. Dans ces conditions, les habitants sont invités à participer à un niveau où les décisions ne se prennent pas ${ }^{9} »$. De plus, nous constatons que l'évolution de l'organisation des pouvoirs locaux se confronte aux considérations des responsables locaux et nationaux convaincus que la proximité entre les institutions et les acteurs suffit à la stimulation de la démocratie. À ce sujet, Loïc Blondiaux rajoute : "L'idée selon laquelle les décisions doivent être prises au plus près de ceux qu'elles touchent est reconnue désormais comme une catégorie générale de l'entendement politique. Les politiques sont invités "à faire proche", dans la posture sinon dans l'action. Dans ce nouvel environnement idéologique, la "démocratie de proximité" s'impose comme une solution naturelle 10 .

Cette recherche de proximité s'est vérifiée dans la définition de nouvelles formes d'intervention visant à créer des articulations systématiques entre les pouvoirs locaux et la participation des habitants. En exemple, la loi du 12

8 CADIOU S., Le pouvoir local en France, Grenoble, PUG, 2006, p. 72.

9 BLONDIAUX L., Le nouvel esprit de la démocratie, Actualité de la démocratie participative, Paris, Seuil, 2008, pp. 67-68.

10 Ibid., p. 66. 
juillet $1983^{11}$ (loi Bouchardeau) renforcée par la loi de 2002 (démocratie et proximité) ont rendu l'enquête publique obligatoire dans certains secteurs (aménagement du territoire, environnement). Une critique essentielle, portée par les militants associatifs ${ }^{12}$, précise que ce dispositif ne prend pas en compte la formation des habitants à la maîtrise des procédures administratives et les connaissances techniques qui englobent les projets. Dans les faits, il est possible de remarquer que ces enquêtes sont souvent lancées à un stade, où les projets sont déjà élaborés, en les considérant comme des procédures, plus qu'une démarche de formation et de consultation des habitants. Dans cette position, les acteurs locaux n'ont que très peu de temps pour engranger les informations et dégager des avis approfondis. De même que leur marge de manœuvre est étroite : la parole des habitants est, dans ces cas-là, prise entre la défense de «l'intérêt général » avancée par les élus et les aspects techniques que les experts sont souvent peu enclins à rendre accessibles.

Le cas de l'intercommunalité nous permet d'avancer qu'il s'agit de lieux de formation au fonctionnement de cette institution. La participation intercommunale nécessite un temps d'apprentissage que les autorités ne prennent pas en compte dans l'organisation des enquêtes publiques ou autres dispositifs. Elle induit aussi l'introduction de nouvelles procédures managériales dans la diffusion et la production de l'information.

La marque de l'autorité n'est plus concentrée dans la décision politique en elle-même mais dans la façon dont elle s'exécute. En outre, les avis défavorables suite à l'établissement des enquêtes publiques sont assez rares et confirment la raison pour laquelle ce dispositif est considéré comme une procédure administrative par les pouvoirs locaux. L'exemple de la construction du stade des Alpes par la communauté d'agglomération Grenoble Alpes Métropole est particulièrement instructif car il permet de vérifier que les logiques politiques et économiques peuvent négliger la législation en matière d'enquête publique. Tout d'abord, un point important et révélateur réside dans le fait que le dépôt du permis de construire (septembre 2002) du stade a précédé la réalisation de l'enquête publique qui, quant à elle, s'est déroulée durant la période septembre-octobre 2002. Cet élément constitue une pratique courante des institutions qui programment l'enquête publique à une période où les orientations majeures ont été prises. Ce positionnement dans le calendrier des procédures est une démarche qui exclut les habitants des débats préalables à la validation des projets, et par là, les prive des informations liées

11 La loi du 12 juillet 1983 assure la démocratisation des enquêtes publiques. Elle préconise que les projets de réalisation d'ouvrages, d'aménagements ou de travaux menés par les collectivités territoriales doivent être précédés d'une enquête publique. Elle précise notamment que l'autorité compétente doit porter à la connaissance du public, par tous moyens appropriés d'affichage, notamment sur les lieux concernés par l'enquête, l'objet de l'enquête, les noms et qualités du commissaire enquêteur ou des membres de la commission d'enquête, la date d'ouverture, le lieu de l'enquête et la durée de celle-ci.

12 Entretien réalisé le 12 juin 2009 avec le responsable de l'antenne grenobloise de l'association Robins des Villes. 
aux négociations économiques et politiques. Ensuite, en plaçant l'enquête publique en dernière étape, les dirigeants locaux présentent un projet détaillé qui ne laisse que peu de place à la révision ou aux changements.

Un rapport commandité par le Ministère de l'Aménagement du Territoire remarque cette pratique et la formule de la façon suivante : "La procédure actuelle des enquêtes publiques intervient à un moment où beaucoup estiment que la question est déjà tranchée. En effet, le projet figure généralement dans des schémas directeurs et des documents d'urbanismes. Il a déjà fait l'objet de décisions administratives. Lorsquil est soumis à consultation, il a déjà atteint un seuil de maturité qui est vécu par le maître d'ouvrage comme un point de non-retour. Or, pendant toute cette phase de maturation le public, malgré les efforts de consultation qui sont pourtant réalisés dans le cadre des documents de planification, a souvent le sentiment de ne pas avoir été associe $e^{-13} »$.

Concernant la construction du stade des Alpes, l'enquête publique initiée par l'autorité préfectorale a tout de même permis de prendre en compte les réserves de certains habitants qui se constitueront en association (SOS Parc Paul Mistral). Face à la négligence et le non-respect, par l'institution intercommunale, des recommandations données par les commissaires (s'appuyant elles-mêmes sur les résultats de l'enquête), la construction du stade sera finalement suspendue pour non-respect des lois concernant la démocratie de proximité le 31 mars $2004^{14}$.

\section{L'organisation de la délibération, une nouvelle compétence intercommunale}

Face à la difficulté à mobiliser des citoyens à travers ces dispositifs et au rôle ambigu des autorités locales, des " acteurs relais » entre l'institution et les habitants ont émergé. La contribution des associations est importante car celles-ci ont participé à construire une méthodologie se dégageant des dispositifs étatiques ou même des services des agences de communication. Durant toute la période des années 1990, un marché s'était présenté pour ces entreprises dans le domaine de la participation. Elles avaient recours à des techniques standardisées reposant sur les enquêtes d'opinions, sondages ou réunions avec un " panel " d'habitants. Elles pouvaient être sollicitées pour des thématiques précises ou, au contraire, dégager des tendances générales de l'opinion locale. Ces techniques ont aussi servi d'outils aux responsables politiques car elles permettaient d'éviter la confrontation avec les acteurs locaux et la diffusion de l'information, en dehors des supports de communication. Du côté des publics, les pratiques suivantes ont alimenté un climat de méfiance car instrumentalisées (destinées à faire la publicité des équipes dirigeantes) dans les discours et supports de communication. Face

13 MÈGRET B., Réforme du système des enquêtes publiques, Synthèse des études réalisées, Paris, Les rapports du Conseil général des ponts et chaussées, 2005, p. 16.

14 Voir article « La petite histoire du stade des Alpes », http://www.letorchondauphinois.fr/-Urbanisme-.html [consulté le 14 juin 2011]. 
à ces actions souvent articulées à des stratégies de conservation du pouvoir, les acteurs locaux ont appris à en décoder les enjeux mais surtout à exiger d'autres dispositifs assurant un échange tourné vers la transparence et les règles démocratiques. Les associations locales ont développé une expertise dans la gestion des dispositifs délibératifs et obtenu des résultats efficaces attirant l'intérêt du pouvoir local. À partir des années 2000, des associations telles que les Robins des Villes ont développé des méthodes de participation en donnant un caractère social à leur activité. Cette association s'illustre par la mise en place de nouvelles techniques d'information et de formation des habitants aux questions d'urbanismes. Par exemple, durant la conduite des projets, elle s'installe dans la zone concernée et entame une campagne d'information (affichage, rencontre avec le conseil de quartier) avant d'organiser des rencontres avec les habitants. Selon l'association, la formation des habitants s'inscrit dans un suivi à long terme, qui, en fonction du type de projet, les prépare autour de quatre niveaux différents de participation : l'information, la consultation, la concertation ou la co-production ${ }^{15}$. Ces méthodes en direction des habitants se fondent notamment sur la formation aux thématiques environnementales et urbanistiques dans le cadre des projets menés par les pouvoirs locaux. L'objectif est de développer l'engagement des habitants dans les projets qui concernent leur cadre de vie et par conséquent, activer une relation sociale avec les techniciens et élus. Il s'avère important de souligner que ces dispositifs, d'un point de vue organisationnel, sont cadrés par l'association ce qui tend à renforcer leur légitimité auprès des habitants. Cette médiation, comme nous avons pu le voir, est souvent voulue par les pouvoirs locaux afin d'anticiper un conflit avec les habitants. C'est pourquoi, ces pratiques sont en quelque sorte dénaturées, dans la mesure où elles ne sont pas considérées comme des procédures se rattachant à une action voulue de discussions démocratiques. Nous pouvons donc établir que ces outils s'associent à une stratégie politique dans de nombreuses situations. Dans le cas de l'intercommunalité, de tels dispositifs ont une fonction essentielle dans le processus de construction territoriale. Ils permettent d'élever la communauté à une fonction d'animateur social et de promouvoir ses projets au niveau local.

En outre, ces outils, lorsqu'ils sont gérés par les pouvoirs politiques, s'adossent nécessairement à la complexité de l'action locale. Le développement des procédures délibératives a davantage jeté le flou sur les pratiques des institutions publiques. Celles-ci ont considéré ces pratiques comme un moyen de renforcer leur légitimité dans les prises de décisions, plutôt que de les intégrer dans le cadre de leur mission publique. Cependant, de nombreuses communautés en essayant de capitaliser les expériences avec des professionnels tels que les agences de communication ou associations, ont mis en place un « Service Concertation » à l'intérieur même de leur organisation.

15 Voir le site de l'association Robins des Villes : http://www.robinsdesvilles.org/spip.php?rubrique1 [consulté le 13 juillet 2011] 
Ces services sont chargés de poursuivre les démarches de concertation sur le territoire mais aussi de favoriser une diffusion de l'information sur l'action intercommunale. La Communauté Urbaine du Grand Lyon ${ }^{16}$, qui avait pour partenaire l'association Robins de Villes dans de nombreuses actions de participation, s'est orientée dans la création d'une Charte de la participation (2003) ainsi que d'un Service Concertation (2003).

Néanmoins, les pratiques de participation sont très hétérogènes. Nous pouvons mettre en exergue trois niveaux d'intégration des procédures délibératives. Elles introduisent de nouvelles procédures organisationnelles entre les services et les dirigeants politiques. Dès lors, nous relevons que les changements organisationnels des institutions sont étroitement reliés aux pratiques sociales des individus.

- une gestion des dispositifs participatifs par l'institution locale à travers une cellule (Service Concertation) chargée du suivi et de l'organisation de ces procédures. Dans ce cas-là, les communautés associent la participation au projet territorial ; celle-ci bénéficie alors d'un financement et de moyens matériels et humains. Elle est donc intégrée à l'organisation intercommunale au même titre que d'autres services. Les procédures délibératives sont placées, dans cette configuration, sous l'autorité de l'équipe dirigeante. L'autonomie de ce service dépend fortement de l'organisation interne de l'institution. Cependant, à l'image du service communication, cet organe peut être placé directement sous l'autorité du directeur de cabinet du Président ou d'un vicePrésident.

- La participation etl'élaboration de procédures délibératives sont soutenues par la structure intercommunale en finançant les acteurs associatifs locaux spécialisés dans ce domaine. Ces associations collaborent avec les institutions locales et exercent une médiation entre les habitants et les pouvoirs locaux. Les collectivités territoriales et structures intercommunales opèrent par des appels d'offres pour soumissionner les associations ou entreprises concernant des actions à mener. Ce type de collaboration favorise une autonomie d'action des acteurs locaux, tout en permettant la constitution d'un réseau local d'acteurs relais de l'intercommunalité.

- Les dispositifs de participation ne s'inscrivent pas dans le projet territorial de la communauté ou ne sont pas perçus comme des outils de gestion que les dirigeants politiques souhaitent rattacher à l'action intercommunale. Ainsi, nous observons que l'action intercommunale est largement déterminée par l'orientation politique de l'équipe dirigeante. Certains courants politiques sont plus sensibles que d'autres à l'instauration des dispositifs délibératifs. Ce rôle d'animation et de concertation peut, en revanche, incomber à la ville centre ou aux communes membres, de façon indépendante. Dans ce cas de

16 Entre 2005-2010, la communauté du Grand Lyon a poursuivi une politique de rénovation urbaine dans de nombreuses zones urbaines créées dans les années soixante (Vénissieux, Vaulx-en-Velin). Durant la poursuite du projet, la communauté a tenté de mobiliser les acteurs locaux autour d'ateliers de discussions. 
figure, il n'y pas une « ingénierie » de la participation ; en cas de crise par exemple les conflits sont gérés sans procédures ou planification.

Grenoble Alpes Métropole, à la période où le Grand Lyon se lança dans une politique de développement des outils participatifs, hésitait encore par la voix du Président de la commission politique, Renzo Sulli, affirmant : "Nous n'avons pas encore tranché la Métro, doit-elle soutenir la participation ou la mettre en cuvre elle-même? L'agglomération n'est pas une supra-communalité, elle ne peut donc rien imposer aux communes. La difficulté réside aussi dans le fait que, plus le niveau de décision est éloigné du citoyen, plus il est difficile de lui faire percevoir qu'il est utile qu'il s'exprime et qu'il s'implique ${ }^{17}$ ».

La question de la participation àl'échelle intercommunale est matériellement difficile à organiser sans la contribution des municipalités. Les structures intercommunales tentent de faire le lien avec les municipalités par le biais des élus communautaires; cependant, le renouvellement des mandats tend à déstabiliser ces actions. Le Service Concertation du Grand Lyon assure une planification des actions selon l'ampleur des projets menés. Concrètement, un calendrier décrivant les étapes de la concertation est diffusé sur le site internet ; la même thématique peut être discutée sur plusieurs lieux de la Communauté Urbaine. Ce service contribue à construire un territoire social et l'identité de l'institution intercommunale. L'engagement de la communauté inclut nécessairement une approche différente de l'action intercommunale pour les dirigeants politiques. Les procédures de participation fournissent des informations sur les attentes des habitants et leur représentation de l'action publique locale. Elle inscrit la politique locale dans le court terme. Ces dispositifs relayent les opinions publiques et certaines décisions sont ainsi prises pour ne pas être en contre-sens des opinions majoritaires au niveau local.

En guise de conclusion, nous nous appuierons sur les travaux de Loïc Blondiaux, sociologue, dont les recherches sont spécialisées dans l'étude des différents dispositifs de participation. Ce chercheur dresse une analyse pertinente des pratiques et conceptions françaises de la participation en employant le terme d' "impératif délibératif ${ }^{18}$ ". Il souligne, à travers son emploi, que le modèle délibératif proposé par Habermas a largement influencé la conception française de la participation provoquant « un changement idéologique ${ }^{19}$ » dans la conduite de l'action publique. Le succès de ce modèle est résumé par Loïc Blondiaux de cette manière : "nous sommes en terme de concepts très pauvres, deux nous servent à penser les mutations en cours : la démocratie représentative nous vivons sous ce régime depuis plus d'un siècle et la

17 BERKANI V., « Participation à l'échelle intercommunale, le grand retard », 2003, Territoires, n441, p. 27. 18 BLONDIAUX L., SINTOMER Y., «L'impératif délibératif », Politix, n57, 2002, pp. 17-35.

19 Ibid., p. 17. 
démocratie participative personne ne sait véritablement ce que c'est, le flou de ce vocable en fait le succès ${ }^{20}$ ".

$\mathrm{Ce}$ «flou » se vérifie dans l'hétérogénéité des pratiques et conceptions autour de la mise en œuvre de ces dispositifs. Il est possible de constater trois difficultés majeures auxquelles sont confrontées les structures intercommunales dans la concrétisation de dispositifs délibératifs. La première difficulté nous permet d'évoquer le «flou des intentions ${ }^{21}$ " qui entoure le recours aux différents dispositifs. Elles peuvent être d'ordre managériale à travers, par exemple, le but de se rapprocher des citoyens ou améliorer les prises de décisions. Les pouvoirs locaux peuvent avoir aussi comme objectif de " réconcilier les citoyens avec la démocratie ». Les contenus des chartes de la participation ou chartes citoyennes, conçues par les communautés et partenaires locaux (associations, partis politiques), explicitent clairement cette ambition.

La deuxième difficulté réside dans «le flou des procédures ${ }^{22}$ »; les agences ou associations spécialisées n'ont pas réussi à «codifier la participation ${ }^{23}$ » selon le sociologue. La diversité des pratiques et leur rattachement à un marché (associations ou agences de communication) ont instauré une dépendance vis-à-vis des institutions locales et limité les innovations comme par exemple celle de Porto Alegre ${ }^{24}$.

La troisième est « le flou des critères d'évaluation ${ }^{25}$ ». Bien qu'Habermas ${ }^{26}$, en développant le modèle délibératif, ait insisté sur la qualité des échanges dans les discussions publiques, il apparaît que ces échanges ne donnent pas lieu précisément à des objectifs : créer du consensus, régler des problèmes, former les citoyens à la démocratie ou promouvoir des projets.

\section{BIBLIOGRAPHIE}

BERKANI V., " Participation à l'échelle intercommunale, le grand retard », Territoires, $n^{\circ} 441,2003$, p. $20-41$.

BLONDIAUX L., " La montée d'un impératif participatif, d'un impératif de concertation » (intervention au colloque de l'Association des Maires des Grandes Villes de France), Territoires, n441, 2003, p. 15-45.

20 BLONDIAUX L. , « La montée d'un impératif participatif, d’un impératif de concertation » (intervention au colloque de l'Association des Maires des Grandes Villes de France), Territoires, n441, 2003, p. 34.

21 Ibid., p. 17.

22 Ibid., p. 36.

23 Ibid., p. 39.

24 Le Parti des Travailleurs (PT) a conquis le pouvoir à Porto Alegre au Brésil en 1989. Pour mettre fin à la corruption, l'administration municipale a travaillé avec des associations afin de définir les orientations budgétaires de la ville. Des instances de prises de décisions composées de délégués élus ont été créées à différents niveaux par exemple 16 forums territoriaux réunissent les délégués de différents quartiers issus de toutes les parties de la ville.

25 Ibid., p. 41

26 HABERMAS J., «Trois versions de la démocratie libérale», Le Débat, n¹25, 2003, p. 125. 
BLONDIAUX L., Le nouvel esprit de la démocratie, Actualité de la démocratie participative, Paris, Seuil, 2008, 109 p.

BLONDIAUX L., SINTOMER Y., " L’impératif délibératif », Politix, n57, 2002, p. 17-35.

CADIOU S., Le pouvoir local en France, Grenoble, PUG, 2006, 206 p.

DEMERS C., «La diffusion d'un changement radical : un processus de redéfinition et de restructuration de l'organisation », Communication E' Organisation, n³, 1993, p. 27-47.

DE CRESCENZO J.-Cl., FLORIS B., " La gestion participative comme de communications organisationnelles ", dans DELCAMBRE P. (dir.), Communications organisationnelles, objet, pratiques, dispositifs, Rennes, PUR, 2000, p. 159-174.

HABERMAS J., "Trois versions de la démocratie libérale», Le Débat, n¹25, 2003, p. 105-135.

LACOSTE M., "Les objets et le travail en collectif », dans DELCAMBRE P. (dir.), Communications organisationnelles, objet, pratiques, dispositifs, Rennes, PUR, 2000, p. 23-33.

MÈGRET B., Réforme du système des enquêtes publiques, Synthèse des études réalisées, Paris, Les rapports du Conseil général des ponts et chaussées, 2005, 156 p.

MIÈGE B., La société conquise par la communication, III. Les Tic entre innovation technique et ancrage social, Grenoble, PUG, 2007, 235 p.

RALLET A., MUSSO P., Stratégies de communication et territoires, Paris, L'Harmattan, 1995, 354 p.

Résumé : Cet article propose une analyse sur l'appropriation des dispositifs délibératifs par les établissements intercommunaux. Il porte un regard critique sur leur intégration dans l'action intercommunale en mentionnant à quels enjeux ils sont associés. Il insiste sur deux points essentiels : l'instrumentalisation de ces dispositifs reliés à des stratégies politiques et les changements organisationnels qu'ils activent dans le fonctionnement de l'institution.

Mots-clés : intercommunalité, délibération, communication, organisation, action publique.

Abstract : The aim of this article is to make an analisis of the ownership of deliberative devices by intercommunal institutions. We try to criticize their integration into the intercommunal action and point out what issues they are associated. We focus on two essentials points: the articulation of these devices to policy strategies and the organizational changes they activate.

Keywords :Intercommunality, Deliberation, Communication, Organization, Public action. 
